\title{
Temperature profiles measurements in turbulent Rayleigh- Bénard convection by optical fibre system at the Barrel of II- menau
}

\author{
Jakub Drahotský1 ${ }^{1 \star}$, Pavel Hanzelka ${ }^{1}$, Věra Musilová ${ }^{1}$, Michal Macek $^{1}$, Ronald du Puits ${ }^{2}$, and Pavel Urban ${ }^{1}$ \\ ${ }^{1}$ Institute of Scientific Instruments of the CAS, v.v.i., Královopolská 147, Brno, Czech Republic \\ ${ }^{2}$ Technische Universität IImenau, Institute of Thermodynamics and Fluid Mechanics, 98684 IImenau, Germany
}

\begin{abstract}
Modelling of large-scale natural (thermally-generated) turbulent flows (such as the turbulent convection in Earth's atmosphere, oceans, or Sun) is approached in laboratory experiments in the simplified model system called the Rayleigh-Bénard convection (RBC). We present preliminary measurements of vertical temperature profiles in the cell with the height of $4.7 \mathrm{~m}, 7.15 \mathrm{~m}$ in diameter, obtained at the Barrel of Ilmenau (BOI), the worldwide largest experimental setup to study highly turbulent RBC, newly equipped with the Luna ODiSI-B optical fibre system. In our configuration, the system permits to measure the temperature with a high spatial resolution of $5 \mathrm{~mm}$ along a very thin glass optical fibre with the length of $5 \mathrm{~m}$ and seems to be perfectly suited for measurement of time series of instantaneous vertical temperature profiles. The system was supplemented with the two Pt100 vertically movable probes specially designed by us for reference temperature profiles measurements.
\end{abstract}

\section{Introduction}

Natural thermally-generated turbulent flows play a vital role in heat transfers in nature, be it the circulation in the atmosphere or the oceans, the flows under the surface of stars or in diverse branches of industry. The RayleighBénard convection (RBC) is a simplified physical model serving for fundamental studies of thermally-generated flows occurring in an Oberbeck-Boussinesq (OB) fluid layer in gravitational field confined between two horizontal, laterally infinite and perfectly conducting plates heated from below and cooled from above [1]. RBC is fully characterized by two dimensionless parameters, the Rayleigh numbers $(R a)$ and the Prandtl number $(P r)$, which are defined as follows: $R a=g(\alpha / v \kappa) \Delta T L^{3}, P r=v / \kappa$. Here $g$ is acceleration due to gravity, $\Delta T$ is the temperature difference between the temperature $T_{b}$ of the bottom plate and temperature $T_{t}$ of the top plate. The plates are mutually separated by the vertical distance $L$. When the experiment is conducted in a vertical cylindrical cell with the diameter $D$, the geometry of the cell is characterised by aspect ratio $\Gamma=D / L$. The physical properties of the fluid in the OB approximation are represented by temperature independent material properties: the isobaric thermal expansivity $\alpha$, the kinematic viscosity $v$, the thermal diffusivity $\kappa$. The temperature variation of density $\rho$ is supposed to be less than a few percent. Breaking these conditions leads to Non-Oberbeck-Boussinesq (NOB) effects.

\footnotetext{
^e-mail: drahotsky@isibrno.cz
}

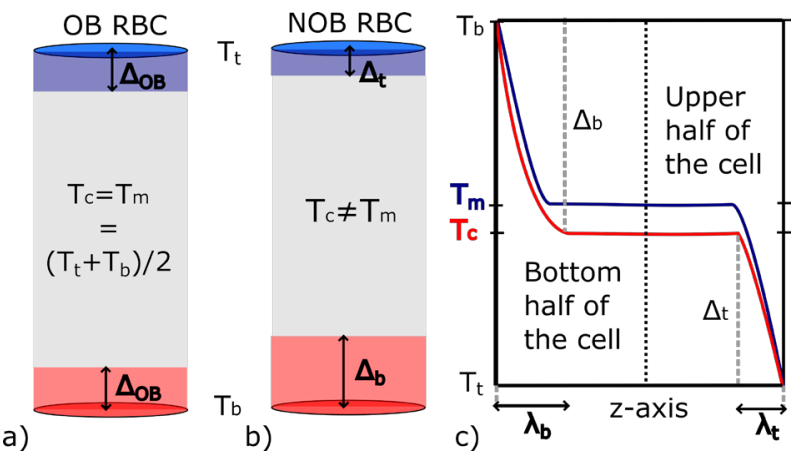

Fig. 1. Schematic illustration of the thermal boundary layers and vertical temperature profiles in RBC: a) Thermal boundary layers in OB RBC where the temperature drops $\left(\Delta_{O B}\right)$ on the top and bottom boundary layers are equal. The turbulent core temperature $T_{c}$ equals the mean temperature $T_{m}$. b) Thermal boundary layers in NOB RBC, where the temperature drops on the top $\left(\Delta_{t}=T_{c}-T_{t}\right)$ and bottom $\left(\Delta_{b}=T_{b}-T_{c}\right)$ layers are asymmetric. The turbulent core temperature $T_{c}$ differs from the mean temperature $T_{m}$. c) The vertical temperature profile in the $\mathrm{OB}$ (blue) and NOB (red) RBC. $\lambda_{t}$ and $\lambda_{b}$ represents the thickness of the top and bottom boundary layers.

The principal motivation of our long-term work is to shed light on the discrepancies among published results on heat transfer efficiency by RBC for $R a$ values above $10^{11}$ [2-4] and influence of NOB effects on RBC, especially on a possible transition of $\mathrm{RBC}$ to the ultimate regime $[5,6]$. It was observed experimentally that NOB effects do lead to asymmetry of the thermal boundary layers adjacent to the 
top and bottom plates (see schematic illustration in Fig. 1), which subsequently alters the heat transfer efficiency at high $R a[5,7]$.

In our previous work, we studied NOB effects in cryogenic RBC system using Czech Cryogenic Turbulence Facility $(\mathrm{CCTF}-3)[8,9]$ with the $\Gamma=1$ cell $(D=L=0.3 \mathrm{~m})$ using cryogenic helium as a working fluid. The current work aims to extend our knowledge about NOB effects in $\mathrm{RBC}$ via measurement of vertical temperature profiles in the cell of $\Gamma \sim 1$ for varying $R a$ numbers in the range of $10^{10}$ to $10^{12}$. The experiments presented here took place in the Barrel of Ilmenau (BOI) [10-12] using dry air as a working fluid. The BOI is newly equipped with the optical fibre system Luna ODISI-B (Optical Distributed Sensor Interrogator) [13] for distributed measurement of temperature along the whole fibre length. In this paper we report the first experiment verifying capabilities of Luna ODISI-B for measurement of temperature profiles in RBC systems.

\section{Experimental arrangement}

\subsection{Barrel of IImenau}

The Barrel of Ilmenau (BOI) is an experimental facility (see Fig. 2) for the study of highly turbulent convection using dry air as a working fluid $(\mathrm{Pr}=0.7$ at atmospheric pressure and working temperatures). The cylindrical cell ( $D=7.15 \mathrm{~m})$ with movable top plate allows to adjust the cell height $L$ in the range of $0.2 \mathrm{~m}$ to $6.3 \mathrm{~m}$. The corresponding $R a$ number and the aspect ratio $\Gamma$ vary in the range of $10^{5}$ to $10^{12}$ and 1.13 to 36 , respectively [10]. The cooling (heating) system of the top (bottom) plate allows to set the temperatures of the plates within the range from $10^{\circ} \mathrm{C}$ up to $80^{\circ} \mathrm{C}$.

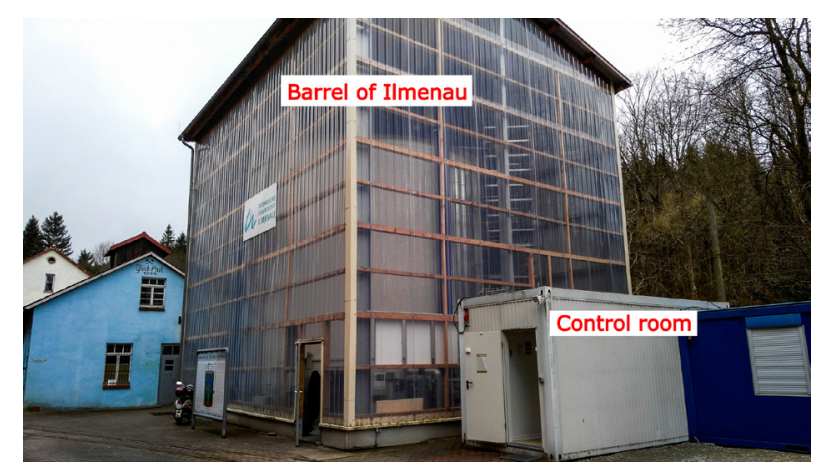

Fig. 2. Experimental facility Barrel of Ilmenau (BOI) is a worldwide largest experiment (diameter $D=7.15 \mathrm{~m}$, adjustable height $L$ up to $6.3 \mathrm{~m}$ ) for the study of highly turbulent convection in dry air.

\subsection{Optical fibre measurement system Luna ODISI-B}

Luna ODISI-B is a system that uses a thin glass optical fibre as a sensor for distributed temperature or strain measurements based on Rayleigh scattering, evaluated by Optical Frequency Domain Reflectometry (OFDR) combined
Table 1. Specifications of the Luna ODiSI-B device installed in the BOI.

\begin{tabular}{|c|c|}
\hline Length of the fibre & $5 \mathrm{~m}$ \\
\hline Temperature repeatability & $\pm 0.4^{\circ} \mathrm{C}$ \\
\hline Temperature range & -268 to $900^{\circ} \mathrm{C}$ \\
\hline Spatial resolution & $5 \mathrm{~mm}$ \\
\hline Acquisition rate & up to $100 \mathrm{~Hz}$ \\
\hline
\end{tabular}

with a Mach Zehnder interferometer [13]. The resulting quantity indicated by the system is called the "frequency shift" that is converted into a temperature information by a suitable calibration (see Sec. 3.2). In general, the system permits to measure the temperature profiles along the fibre with the length of up to $20 \mathrm{~m}$ with acquisition rate of $250 \mathrm{~Hz}$ and spatial resolution down to $1.25 \mathrm{~mm}$ in dependence on type of the system configuration. Detailed parameters of the system used in our experiments are given in Table 1.

The installation of Luna ODISI-B together with Pt100 probes in the BOI is schematically shown in Fig. 3. The fibre is fastened to a special holder above the top plate, passes through a hole in the plate down to the bottom plate where it is fixed. The last $\sim 10 \mathrm{~cm}$ of the fibre cannot be used for the measurements (because of the low refraction termination). To obtain also temperature profile within the region of the bottom boundary layer, we bent this part of the fibre and fixed it to the bottom plate.

The temperature profiles were measured at two verticals: along the cell axis and a vertical line displaced $1 \mathrm{~m}$ from the sidewall (side position). The arrangement of the Luna ODISI-B system above the top plate for measurements at the central axis is shown in Fig. 4.

The thermal response of the fibre is dominated mainly by $\mathrm{d} n / \mathrm{d} T$ effect, where $n$ is the index of refraction and $T$ is the temperature. To fix the fibre in the defined position, we glued it to a supporting fishing line with little bends by epoxide every $30 \mathrm{~cm}$. The fishing line was vertically tightened between the plates. We suppose that in this configuration, the deformation of fibre and its effect on measured profiles is minimized.

\subsection{Pt100 temperature probes for reference temperature profiles measurements}

The Luna ODISI-B system was supplemented with two vertically movable Pt100 probes specially designed at the Institute of Scientific Instruments of the CAS (ISI) for reference measurements of the temperature profiles (Fig. 5). The lower Pt100 probe was used for the measurement in the region of the bottom boundary layer and turbulent core, the upper Pt100 probe was used only for measurement in the region of the top boundary layer (Fig. 3).

Both Pt100 probes were suspended on the feed cables passing through the hole in the top plate. We could move the probes by hand via cables up and down. 


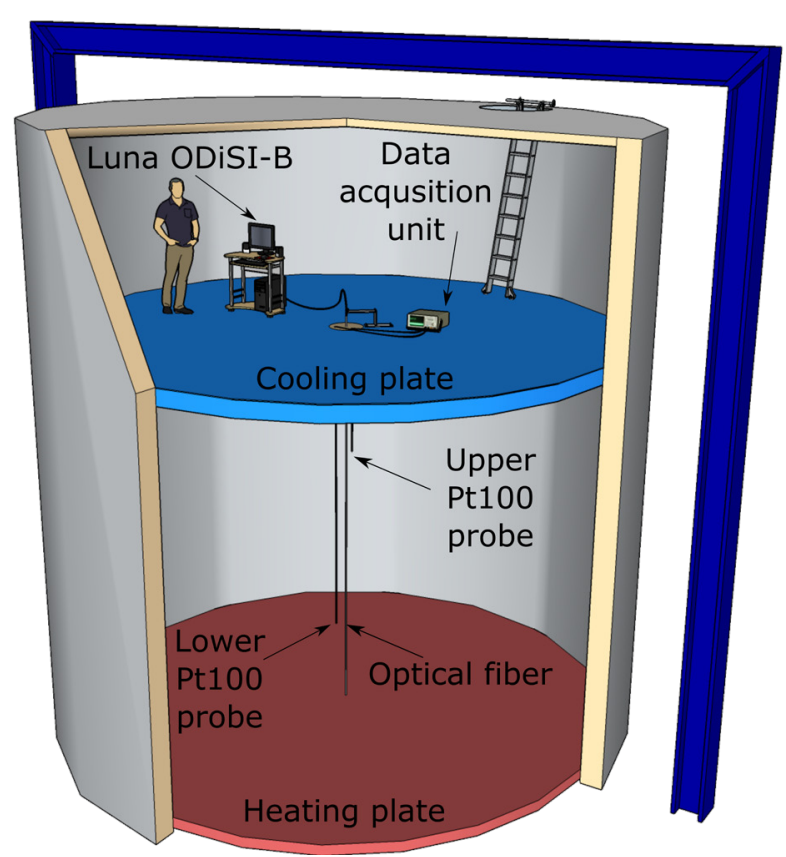

Fig. 3. Schematic illustration of Luna ODISI-B and Pt100 probes installation in the axis of BOI. Luna ODiSi-B system and data acquisition unit for Pt100 probes were placed on an upper side of the top plate. The optical fibre passes through a hole in the plate and is fixed to the bottom plate. Both Pt100 probes were suspended on the feed cables passing through the hole in the top plate together with the fibre. The upper Pt100 probe serves only for measurement of temperatures profiles across the top boundary layer, whereas the lower Pt100 probe serves for measurement both across the bottom layer and in the turbulent core.

The Pt100 probes consist of a $0.3 \mathrm{~mm}$ thin platinum resistor film (type 03002 , tolerance class A with $0.25^{\circ} \mathrm{C}$ uncertainty at $45^{\circ} \mathrm{C}$ ) produced by ZPA EKOREG [14]. The resistors with the nominal resistance value of $100 \Omega$ (measurement range of $-40{ }^{\circ} \mathrm{C}$ to $400{ }^{\circ} \mathrm{C}$ ) and nominal size of $2.3 \mathrm{~mm} \times 5.0 \mathrm{~mm}$ is fastened to a small brass plate with a gold tip. The brass plate $(0.7 \mathrm{~mm}$ in thickness $)$ is linked to the $1.5 \mathrm{~mm}$ thick rectangular holder $(6 \mathrm{~mm} \times 250 \mathrm{~mm})$ made of fibreglass. The sensor is connected to the copper contacts of a cable connector via 4-wire "ribbon cable" Lake Shore WQL 36 composed of thin phosphor bronze wires (Fig. 5). The gold tip works as an electrical indicator of a mechanical contact of the probe with the bottom plate and it is also electrically connected to the cable connector. To prevent swinging of the lower Pt100 probe on the $4.7 \mathrm{~m}$ long feed cable, we equipped the holder with two guide handles sliding along a fishing line vertically tightened between the plates. Moreover, an additional weight in the form of a brass ring was placed on the lower probe. This weight keeps the feed cable tight. The materials chosen for manufacturing of the probes have low thermal conductivity with the aim to minimize the influence of the probes on the measured temperature profiles.

\section{Results and discussions}

We performed the test measurements of vertical temperature profiles by Luna ODISI-B and reference Pt100 probes

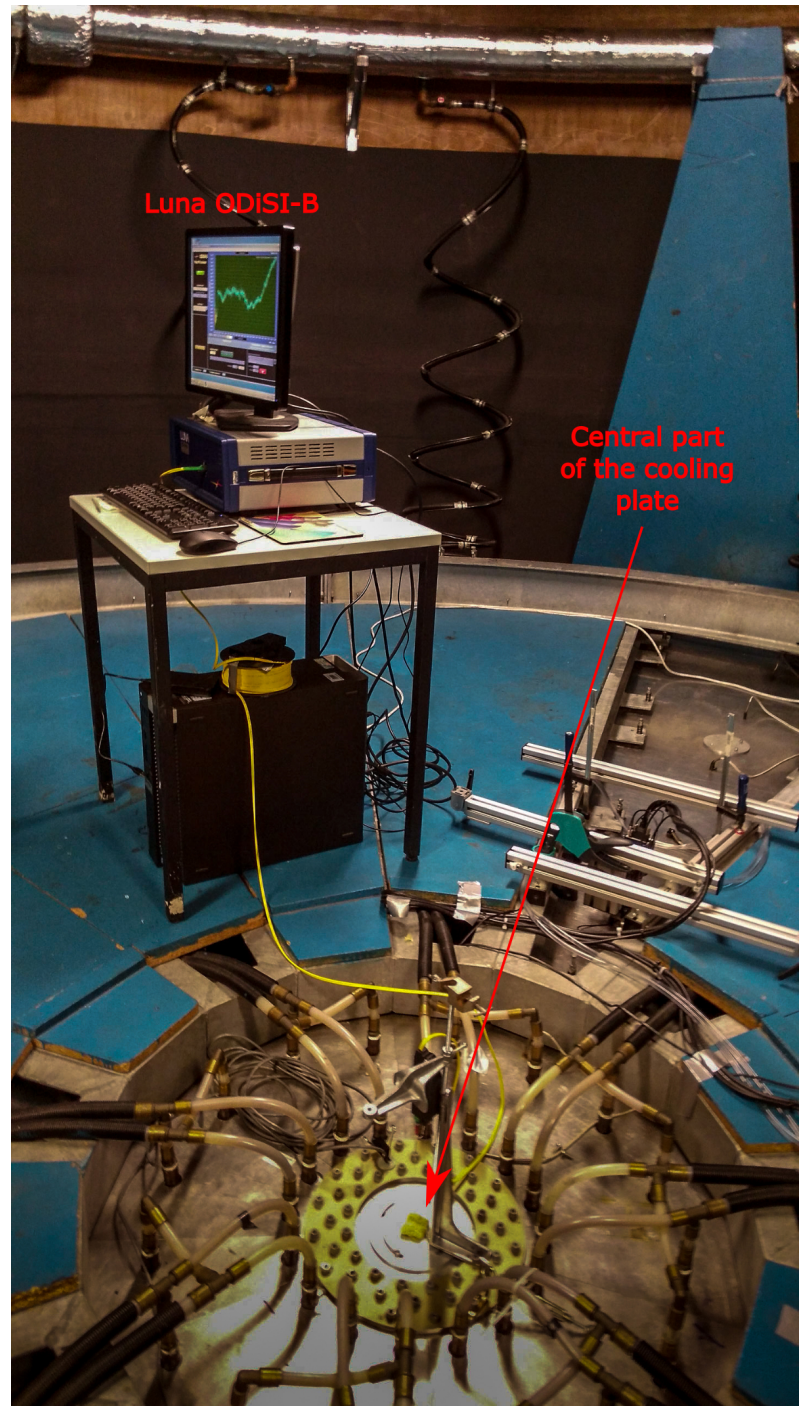

Fig. 4. The arrangement of Luna ODISI-B in the space above the top plate. Optical fibre here enters the cell in the centre of the plate. The pipelines belong to the water cooling system of the plate.

along (i) the cell axis and (ii) along a vertical at a radial position of $1 \mathrm{~m}$ from the cell sidewall. The measurements were done for various temperature differences $\Delta T$ between the plates within the range of $R a$ numbers of $1 \times 10^{10}$ to $4 \times 10^{11}$. In all measurements, the cell was filled with dry air at atmospheric pressure. The mean temperature $T_{m}$ of air was kept at $30{ }^{\circ} \mathrm{C} \pm 0.1{ }^{\circ} \mathrm{C}$. In the following sections (3.1 - 3.3) we present example test measurements obtained at two $R a$ numbers.

\subsection{Temperature profiles measured by Pt100 probes}

Time-averaged temperature profiles $\left\langle T_{P t}(z)\right\rangle$ measured by Pt100 probes in both locations along the central axis and out of the centre are presented in Fig. 6. The $\left\langle T_{P t}(z)\right\rangle$ profiles represent time-averaged temperatures measured in each distance $z$ within 1 minute interval with the acquisition rate of $10 \mathrm{~Hz}$. The profiles were measured at $R a=2.18 \times 10^{11}$ with the temperature difference of $\Delta T=$ 
$20^{\circ} \mathrm{C}$. In the region of the boundary layers, the measurements were done with steps of $5 \mathrm{~mm}$. Due to the dimensions of the probes, it was not possible to obtain temperatures closer than $5 \mathrm{~mm}$ from the plates. Although only few points could be measured directly in the boundary layers, the transitions of temperature profiles from the boundary

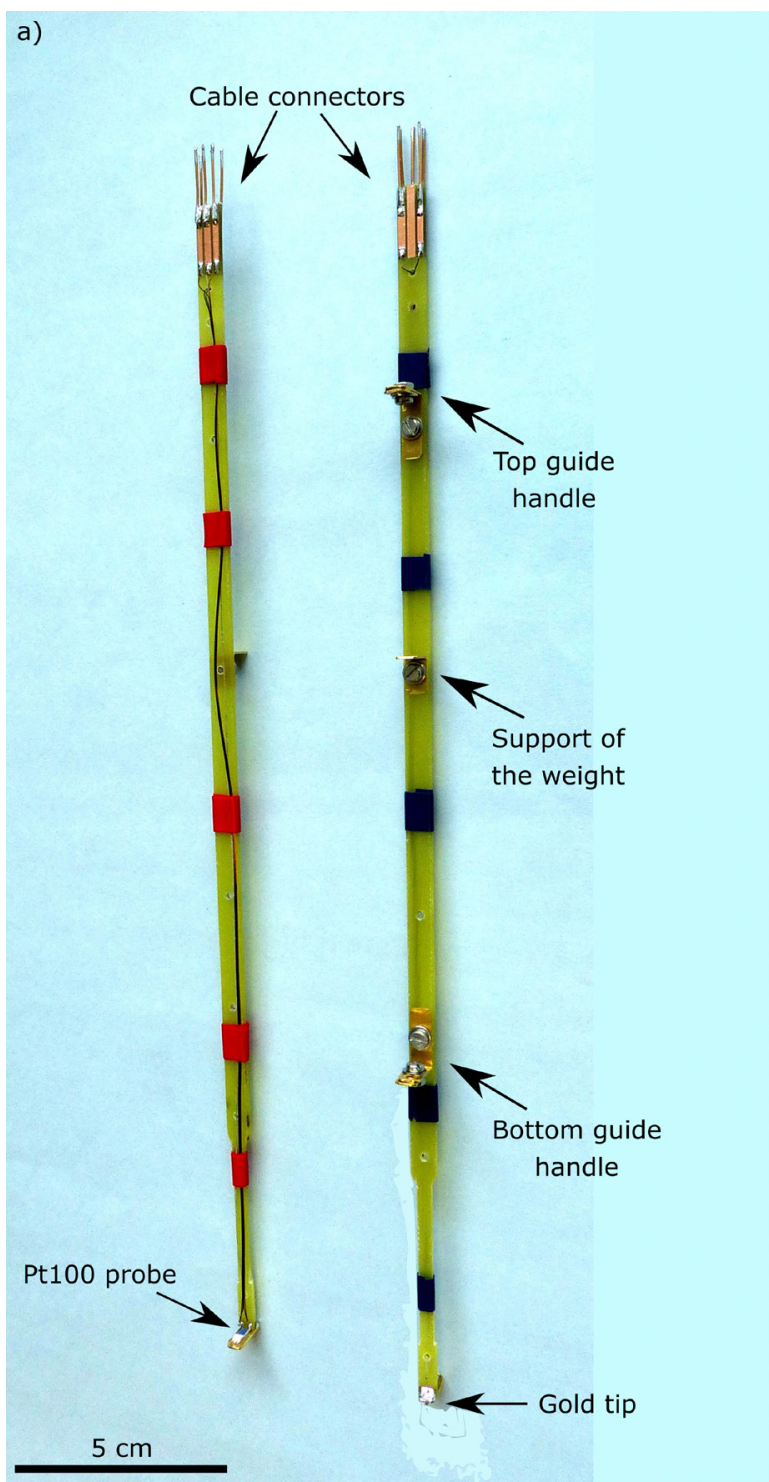

b)

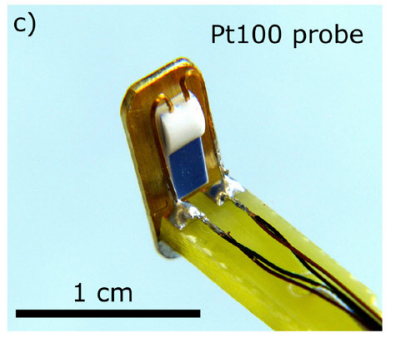

Fig. 5. Lower Pt100 probe for the reference temperature profiles measurements designed at ISI. a) The probe is shown from the front and back side. b) Detail view on of the gold tip which serves as the electrical indicator of mechanical contact of the probe with the bottom plate. Opposite to the tip, the small electrode made of the copper sheet is installed on the surface of the bottom plate to secure the electrical connection. c) Detail view of the Pt100 sensor fastened to the brass plate. layers to the bulk are clearly visible. The shift of the side position profile to higher temperatures is expected as a result of upward convection flow near the side wall. The differences between the time-averaged temperatures $\left\langle T_{P t}(z)\right\rangle$ and the mean temperature $T_{m}$ in the region of turbulent core can be ascribed to the NOB effects $[3,5]$. The situation corresponds to the schematic illustration in Fig. 1b, however with the BL asymmetry inverted (the measured bottom BL is in fact narrower than the top BL).

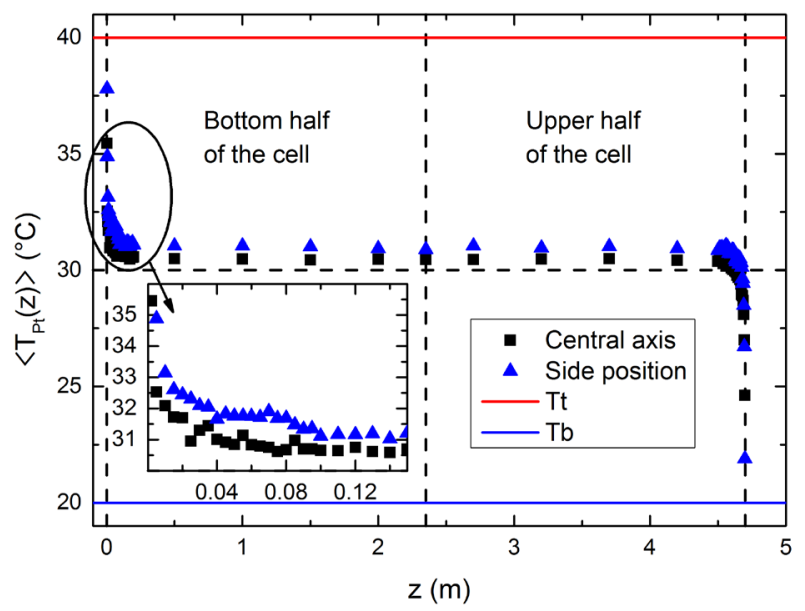

Fig. 6. Time-averaged temperature profiles as $\left\langle T_{P_{t}}(z)\right\rangle$ a function of distance $z$ from the bottom conducting plate measured by Pt100 probes $\left(\Delta T=20^{\circ} \mathrm{C}, R a=2.18 \times 10^{11}\right)$. The vertical dashed black lines indicate the location of the cell centre and the plates position. The blue filled triangles represent the measurements along the central axis of the cell, the black filled squares represent the measurements along the axis $1 \mathrm{~m}$ away from the cell sidewall. The inset shows detail view on the profiles in the region of the bottom boundary layer. The solid horizontal lines represent the mean temperatures of the top $\left(T_{t}\right.$ - blue line) and bottom $\left(T_{b}-\right.$ red line $)$ plates.

\subsection{Calibration of Luna ODISI-B}

Fig. 7 shows the measured time-averaged frequency shift as a function of distance $z$ away from the bottom plate at $\Delta T=30^{\circ} \mathrm{C}\left(R a=3.27 \times 10^{11}\right)$. The data correspond to an average obtained from a one and half hour long measurement with the acquisition rate of $10 \mathrm{~Hz}$. The inset shows a calibration curve used for the transformation of the time-averaged frequency shift onto the time-averaged temperature $\langle T(z)\rangle$. The red solid curve is a second order polynomial fit of the three selected data points at the plates and in the centre of the cell. Temperatures used for calibration curve were measured by the lower Pt100 probe moved to the centre of the cell and by temperature sensors embedded in the plates. Minimal (maximal) frequency shift was assigned to the temperature of the bottom heating (top cooling) plate.

\subsection{Temperature profiles measured by Luna ODISI-B}

Resulting time-averaged temperature profile $\langle T(z)\rangle$ is shown in Fig. 8 (green solid line and filled green circles). 


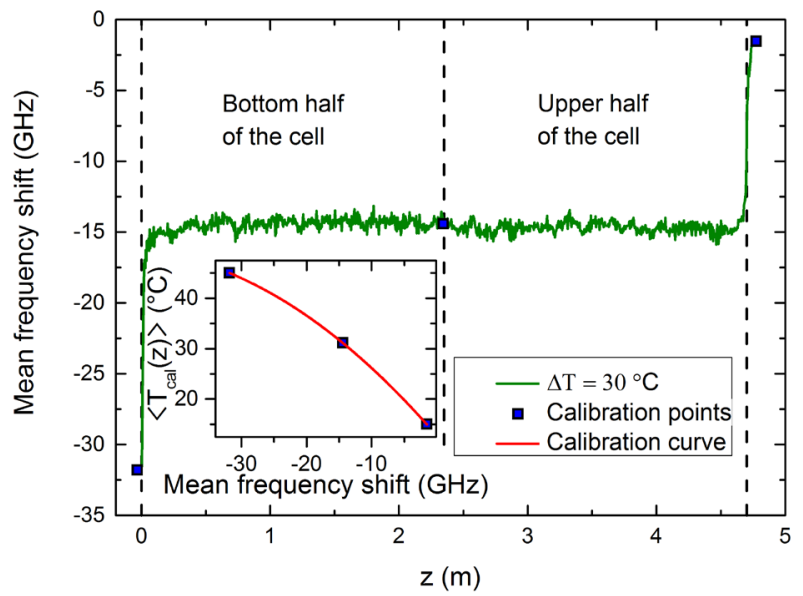

Fig. 7. The time-averaged frequency shift as a function of the vertical distance $z$ from the bottom conducting plate $(\Delta T=$ $30^{\circ} \mathrm{C}, R a=3.27 \times 10^{11}$ ) measured in a vertical $1 \mathrm{~m}$ from the cell sidewall. The vertical dashed black lines indicate the location of the cell centre and the positions of the plates. The inset represents a calibration curve used for transformation of the time-averaged frequency shift to the time-averaged temperature $\langle T(z)\rangle$. The red curve is second order polynomial fit of the three selected data points shown as blue squares. The temperature $\left\langle T_{c a l}(z)\right\rangle$ of the middle point was measured by the lower Pt100 probe, the other two points were measured by sensors placed in plates.

Here $\langle T(z)\rangle$ profile is compared with the profile measured by Pt100 probes (blue filled triangles). The detailed view on the profiles in the region of the boundary layer at the bottom plate shows that the both profiles do not fit one to each other ideally (see inset). It is probably caused by imperfect calibration curve which must be refined in the future. In addition, the correction of the background spatial noise pattern $\sim \pm 1{ }^{\circ} \mathrm{C}$, due to fibre imperfection (seen now in Figs. 7 and 8) needs to be done.

\section{Conclusions}

We applied a new optical system LUNA ODiSI-B and presented here preliminary results on vertical temperature profiles measurement in turbulent RBC across the whole height of the experimental cell, involving the turbulent core as well as the thermal boundary layers. This system, using a glass optical fibre, has not yet been used in any RBC experiments. The LUNA ODiSI-B should enable us to get instantaneous profiles with a high spatial resolution of about $5 \mathrm{~mm}$ along the fibre with the length of $4.7 \mathrm{~m}$. We compared the time averaged profiles obtained by this method and by movable Pt100 temperatures probes. The temperatures obtained by Luna ODISI-B with calibration curve delivered by the manufacturer failed in our measurements. Therefore we performed our own simple three point calibration before each of the measurement for various $R a$ numbers. The evaluated temperature profiles within boundary layers still differed markedly from the profiles obtained by Pt100 probes. The calibration will be refined in the future data processing, and corrections extracting the background spatial noise due to fibre imperfection will be done. Reference temperature profiles were

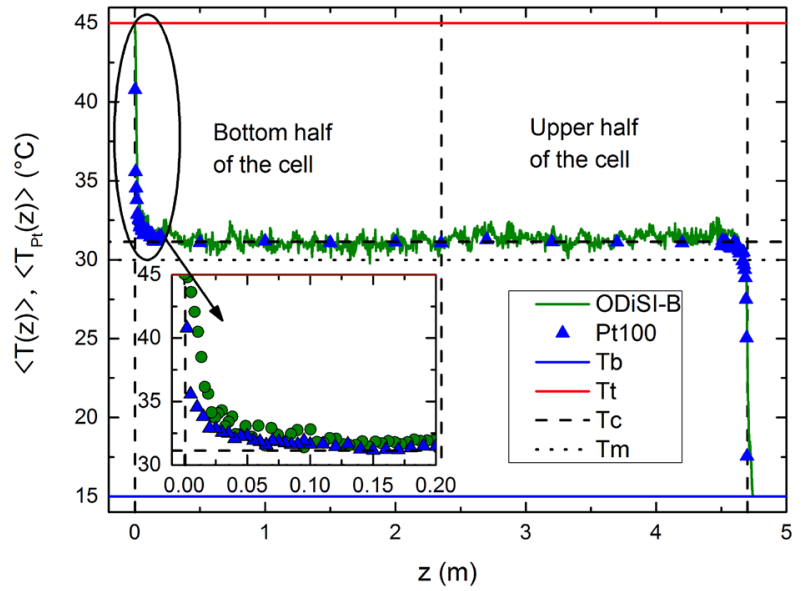

Fig. 8. Comparison of the time-averaged temperatures measured by Luna ODISI-B (green solid curve, filled green circles) and Pt100 probes (blue filled triangles) as a function of distance $\mathrm{Z}$ from the bottom plate $\left(\Delta T=30^{\circ} \mathrm{C}, R a=3.27 \times 10^{11}\right)$. Both profiles were measured along the vertical in the radial position $1 \mathrm{~m}$ from the cell sidewall. The vertical dashed black lines indicate the location of the cell centre and the plates. The solid horizontal lines represent the time-averaged temperatures of the top $\left(T_{t}\right.$ blue line) and bottom ( $T_{b}$ - red line) plates. The horizontal dotted black line corresponds to the time-averaged mean plate temperature $T_{m}$. The dashed black line represents the time-averaged turbulent core temperature $T_{c}$ measured in the cell centre by Pt100 probe.

obtained by Pt100 probe measurement. In these, asymmetry between top and bottom thermal boundary layers (NOB effect) was observed. Opposite to Pt100 probes, the Luna ODISI-B is promising for study of the time-dependence [15] of temperature profiles and space-time temperature correlations [16]. This motivates us to continue the study of applicability of this method in RBC experiments.

\section{Acknowledgements}

We thank L. Skrbek, T. Králík, and A. Srnka for stimulating discussions, and further especially to P. Krejčí for technical support with the Pt100 probes manufacturing and S. Abawi and V. Mitschunas for help with performing the measurements at BOI. We acknowledge the support of this research by the Czech Science Foundation under the project GA17-03572S and by the European Community Framework Programme 7, EuHIT (European Highperformance Infrastructures in Turbulence) Grant Agreement No. 312778. M. Macek acknowledges the support by the MEYS CR project LO1212.

\section{References}

1. G. Ahlers, S. Grossmann, D. Lohse, Rev. Mod. Phys., 81, 503 (2009)

2. F. Chillà and J. Schumacher, Eur. Phys. J. E, 35, 58 (2012)

3. P. Urban, P. Hanzelka, V. Musilová, T. Králík, M. La Mantia, A. Srnka, L. Skrbek, New J. Phys., 16, 053042 (2014) 
4. P. Urban, V. Musilová, L. Skrbek, Phys. Rev. Lett., 107, 014302 (2011)

5. L. Skrbek and P. Urban, J. Fluid Mech., 785, 270 (2015)

6. X. He, E. Bodenschatz, G. Ahlers, J. Fluid Mech., 791, R3 (2016)

7. P. Urban, P. Hanzelka, T. Králík, V. Musilová, A. Srnka and L. Skrbek, Phys. Rev. Lett., 109, 154301 (2012)

8. P. Urban, P. Hanzelka, T. Králík, V. Musilová, L. Skrbek, and A. Srnka, Rev. Sci. Instrum., 81, 085103 (2010)

9. https://www.euhit.org/infras/czech-cryogenicturbulence-facility/facilities
10. R du Puits, Ch. Resagk and A. Thess, New J. Phys., 15, 013040 (2013)

11. R du Puits, Ch. Resagk, A. Tilgner, F. H. Busse, A. Thess, J. Fluid Mech., 572, 231 (2007)

12. https://www.euhit.org/infras/barrel-of-ilmenau

13. http://lunainc.com/odisi

14. http://www.zpaul.cz/

15. Y. Wang, X. He, P. Tong, Phys. Rev. Fluids, 1, 082301(R) (2016)

16. V. Musilová, T. Králík, M. La Mantia, M. Macek, P. Urban, L. Skrbek, J. Fluid. Mech. 832, 721 (2017) 